\title{
论 文＼cjkstart食品安全检测新技术与新方法专刊
}

\section{QuEChERS 预处理结合 HPLC-MS/MS 同时检测 茶叶中 7 种农药残留}

陈䂞 ${ }^{\circledR 2}$ ，上官良敏 ${ }^{(3)}$, 付风富 ${ }^{(2 *}$

(1) 上海市农业科学院农产品质量标准与检测技术研究所, 上海 201403

(2) 福州大学食品安全分析与检测教育部重点实验室; 海西食品安全检测技术与产品协同创新中心, 福州 350108

(3) 国家茶叶质量监督检验中心(福建), 安溪 362400

*通讯作者, E-mail: fengfu@fzu.edu.cn

收稿日期: 2015-09-14; 接受日期: 2015-10-30; 网络出版日期: 2016-01-27

福建省自然科学基金项目资助(编号: 2012J01035)

摘要本文建立了同时分析检测茶叶中啶虫脒、吡虫啉、噻虫嗪、多菌灵、克百威、灭多威和丁醚腿 7 种农 药残留的 QuEChERS 前处理结合高效液相色谱-质谱联用(QuEChERS-HPLC-MS/MS)技术. 优化了 QuEChERS 前处理的各种条件和 HPLC-MS/MS 分离检测条件. 所建立的方法简单、快速, 可用于同时分析检测茶叶中上述 7 种农药残留, 效果良好. 7 种农药的国际纯粹与应用化学联合会(IUPAC)定量限为 $0.26 \sim 1.3 \mathrm{ng} / \mathrm{mL}$, 回收率为 $86 \%$ 101\%, 能够满足国内外茶叶标准对其限量指标的检测要求.

关键词 QuEChERS, HPLC-MS, 茶叶, 氨基甲酸酯农药, 新烟碱类农药, 丁醚脲

\section{1 引言}

氨基甲酸酯类农药多菌灵、灭多威和克百威是广 谱杀虫剂, 作为高毒有机氯农药的替代品, 曾广泛应 用于茶叶种植中. 虽然它们的毒性较低, 但是过量摄 入仍会对人体健康造成一定的危害 ${ }^{[1 ~ 3]}$. 美国环境保 护署(EPA)已将多菌灵、灭多威和克百威列入优先控 制目录 ${ }^{[4]}$, 我国农业部于 2002 年发布公告, 禁止克百 威在茶树上使用 ${ }^{[5]} .2011$ 年农业部也撤销了灭多威在 茶树上的使用登记. 世界卫生组织(WHO)甚至将灭 多威定义为剧毒农药 ${ }^{[6]}$. 虽然我国已明文禁止克百威 和灭多威在茶叶种植中的使用, 但是由于其高效的
灭虫效果, 目前仍有部分茶农在利益的驱使下违规 使用. 新烟碱类农药吡虫啉、啶虫脒和噻虫嗪对昆虫 广普有效, 具有良好的持久性 ${ }^{[7]}$. 近年来这 3 种农药 在我国茶叶生产中应用较广, 用以防治小绿叶蝉、飞 禹、蓟马、黑刺粉虫等害虫 ${ }^{[8 \sim 11]}$. 目前在我国已经有 大量的茶园使用新烟碱类杀虫剂进行病虫害防治. 新烟碱类农药中吡虫啉被 WHO 和 EPA 列为 II 类中 等毒性 ${ }^{[12]}$, 具有细胞毒性, 且对人体外周血淋巴细胞 遗传物质有损伤作用 ${ }^{[9]}$. 噻虫嗪属低毒杀虫剂, 但其 降解产物的毒性尚不清楚 ${ }^{[13]}$. 新型硫艮类农药丁醚 脲是选择性杀虫和杀螨剂，广泛用于茶树上，对蚜 虫、叶蝉、粉闽、蛾和螨类具有很好的杀灭效果. 丁

\footnotetext{
引用格式: 陈磊, 上官良敏, 付风富. QuEChERS 预处理结合 HPLC-MS/MS 同时检测茶叶中 7 种农药残留物. 中国科学: 化学, 2016, 46: 302-308 Chen L, Shangguan LM, Fu FF. Simultaneous determination of seven carbamates, neonicotinoids and diafenthiuron pesticide residues in tea by Chen L, Shangguan LM, Fu FF. Simultaneous determination of seven carbamates, neon
QuEChERS-HPLC-MS/MS. Sci Sin Chem, 2016, 46: 302-308 doi: 10.1360/N032015-00188
} 
醚脲代谢物比原药本身具有更高的毒性, 因此, 2002 年欧盟对丁醚艮实行禁用、禁售 ${ }^{[14]}$.丁醚嫝经长时间 曝晒后, 其蒸发和分解产物对肺部有积累性的毒性 作用 ${ }^{[15,16]}$. 目前, 欧盟对茶叶中吡虫啉 (IMID)、啶虫 脒 (ACET)、噻虫嗪(THIA)、多菌灵(CARBE)、克百 威(CARBO)、灭多威(METH)和丁醚脲(DIAF) 7 种 农药的最大残留限量规定分别为 $0.05 、 0.1 、 20 、 0.1$ 、 $0.05 、 0.1 \mathrm{mg} / \mathrm{kg}$ 和不得检出 ${ }^{[17]}$, 所以对茶叶中的多菌 灵、灭多威、克百威、吡虫啉、啶虫脒、噻虫嗪和丁 醚脲残留进行检测具有重要意义.

目前，茶叶中氨基甲酸酯类农药、新烟碱类农药 和丁醚脲残留检测的样品前处理方法主要有固相萃 取(SPE)、固相微萃取(SPME)、加速溶剂萃取(ASE) 和基质固相分散(MSPD) 等 ${ }^{[15,18 ~ 20]}$. 这些方法成本较 高, 而且前处理过程繁琐、耗时. QuEChERS (quick, easy, cheap, effective, rugged, safe)前处理方法, 适用于 不同种类农药的同时检测，已成为一种重要的农残前 处理方法. QuEChERS 方法已用于茶叶 ${ }^{[21 ~ 24]}$ 、植物性 食品(水果、蔬菜) ${ }^{[25 ~ 31]}$ 、土壤 ${ }^{[32,33]}$ 等样品的前处理, 一 般用乙腈或含 $1 \%$ 乙酸的乙腈溶液提取, 加入硫酸镁、 氯化钠或醋酸钠的组合除水, 无水硫酸镁、乙二胺 $N-$ 丙基硅烷(PSA)、石墨化炭黑(GCB)或 $\mathrm{C}_{18}$ 的组合净化, 该方法操作简单、快速, 精密度和回收率较好. 目前, 还没有有关 QuEChERS 法同时富集处理茶叶中吡虫 啉、啶虫脒、噻虫嗪、多菌灵、克百威、灭多威和丁 醚嫝 7 种农药的报道. 本文的主要目的是开发一种基 于 QuEChERS 前处理技术结合 HPLC-MS/MS, 同时分 析检测茶叶中 7 种氨基甲酸酯类、新烟碱类和新型硫 脲类农药残留的高灵敏、快速检测方法, 以期能更加 快速、简易、廉价和准确地评价茶叶的质量安全.

\section{2 实验部分}

\section{1 试剂与仪器}

本实验所用的仪器为 Agilent 1290/6410 高效液 相色谱-三重四级杆质谱联用仪(HPLC-MS/MS, USA), 配有 Zorbax SB-C 18 分离柱 $(2.1 \mathrm{~mm} \times 150 \mathrm{~mm}, 3.5 \mu \mathrm{m})$. $100 \mu \mathrm{g} / \mathrm{mL}$ 的啶虫脒、吡虫啉、噻虫嗪、多菌灵、灭 多威、克百威和丁醚脲(图 1)标准溶液购自农业部环 境保护科研监测所, 保存在 $-5^{\circ} \mathrm{C}$ 环境下. 更低浓度的 标准溶液由上述标准溶液用乙腈-水 $(1: 1)$ 逐步稀释而 成, 并保存在 $-5^{\circ} \mathrm{C}$ 条件下, 保质期 1 个月. 乙腈(色谱 纯)、甲酸(色谱纯)、甲苯和氯化钠(分析纯)均购自国
药集团有限公司 (中国). $N$-丙基乙二胺(PSA，40 63 $\mu \mathrm{m}, 50 \mathrm{~g} /$ 瓶)和石墨化炭黑(GCB, 120 400 目, $50 \mathrm{~g} /$ 瓶) 均产于上海安谱科学仪器有限公司(中国). $0.22 \mu \mathrm{m}$ 针 筒式滤膜过滤器由天津津腾实验设备有限公司 (中国) 生产. 实验所用的水是 Milli-Q 水(18.2 M $\Omega \mathrm{cm})$.

\section{2 实验方法}

称取粉碎后的茶叶样品 $2.0 \mathrm{~g}$, 放入 $50 \mathrm{~mL}$ 的离 心管中, 加入 $10 \mathrm{~mL}$ 的乙腈, $0.5 \mathrm{~g}$ 的 $\mathrm{NaCl}$, 漩浴混匀 $1 \mathrm{~min}$ 后, 在 $50^{\circ} \mathrm{C}$ 水浴条件下, 超声提取 $10 \mathrm{~min}$. 然 后, 在 $4000 \mathrm{r} / \mathrm{min}$ 下离心 $5 \mathrm{~min}$, 取 $1.5 \mathrm{~mL}$ 上层澄清 液放入 $10 \mathrm{~mL}$ 的离心管中, 加入 $0.15 \mathrm{~g} \mathrm{~N}$-丙基乙二 胺、 $0.15 \mathrm{~g}$ 石墨化炭黑及 $0.5 \mathrm{~mL}$ 甲苯溶液, 漩浴混匀 $1 \mathrm{~min}$ 后, 在 $4000 \mathrm{r} / \mathrm{min}$ 下离心 $5 \mathrm{~min}$, 取上清液过 $0.22 \mu \mathrm{m}$ 滤膜, 滤液用于 HPLC-MS/MS 测定 ${ }^{[34]}$. HPLC-MS/MS 条件如下: 柱温 $30^{\circ} \mathrm{C}$, 流动相 $\mathrm{A}$ 为 $0.1 \%$ 甲酸水溶液 $(V / V)$, 流动相 $\mathrm{B}$ 为乙腈, 流动相流 速为 $0.2 \mathrm{~mL} / \mathrm{min}$, 进样量为 $10 \mu \mathrm{L}$. 梯度洗脱程序如 下: 0 12 min, B 相的比例从 5\%逐步升高到 $90 \%$, 维 持 $3 \mathrm{~min} ; 15 \sim 17 \mathrm{~min}, \mathrm{~B}$ 相比例从 $90 \%$ 逐渐减小到 5\%, 维持 $3 \mathrm{~min} . \mathrm{ESI}(+)$ 扫描方式为多反应监测 $(\mathrm{MRM})$, 电 喷雾电压 $4000 \mathrm{~V}$, 离子源温度 $300^{\circ} \mathrm{C}$, 雾化气压力 35 psi, 最佳的定量、定性离子等参数见表 1 .

表 1 在 HPLC-MS/MS 上 7 种待测农药的主要检测参数

\begin{tabular}{cccc}
\hline 待测农药 & 分子量 & 母离子 $(\mathrm{m} / \mathrm{z})$ & $\begin{array}{c}\text { 定量/定性离子 } \\
(\mathrm{m} / \mathrm{z})\end{array}$ \\
\hline 啶虫缕 & 222.8 & 223.2 & $126^{*}, 56.1$ \\
多菌灵 & 191.2 & 192.1 & $160.1^{*}, 132$ \\
丁醚脲 & 384.6 & 385 & $329.1^{*}, 278.2$ \\
噻虫嗪 & 291.7 & 292.1 & $211^{*}, 181$ \\
吡虫啉 & 255.7 & 256.2 & $209^{*}, 175$ \\
克百威 & 221.3 & 222.1 & $123^{*}, 165.1$ \\
灭多威 & 162.2 & 163.1 & $106^{*}, 88.1$ \\
\hline
\end{tabular}
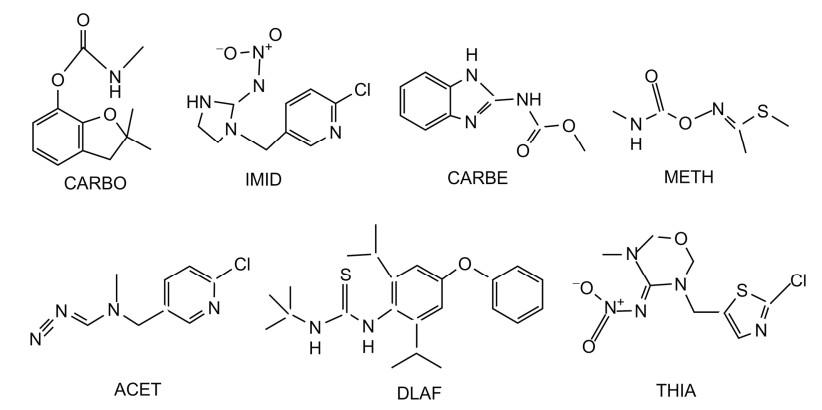

图 17 种待测农药的化学结构 


\section{3 结果与讨论}

\subsection{QuEChERS 法提取及其优化}

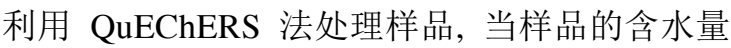
小于 $25 \%$ 时, 通常需要在处理过程中适当补加水. 王 翔等 ${ }^{[35]}$ 详细研究了加水浸泡对于干茶叶中农残提取 的影响, 结果表明, 加水浸泡反而降低干茶叶样品中 农残的提取率. 所以, 本文在提取过程中不加水, 直 接采用乙腈作为提取溶剂提取干茶叶中的啶虫脒、噻 虫嗪、吡虫啉、多菌灵、灭多威、克百威和丁醚脲. 在 QuEChERS 法提取过程中, 其他物质, 如色素和多酚 类物质等也会被提取出来. 这些物质的存在会严重 影响随后 HPLC-MS/MS 检测的灵敏度、准确度和精 确度. 为了保证待测农药完全被提取出来，同时最大 限度地抑制色素和多酚类等干扰物质的提取，我们 对剂料比(提取溶剂体积和茶叶样品质量的比例)进 行了详细优化. 按照 $3: 1 、 4: 1 、 5: 1 、 6: 1 、 7: 1$ 和 $8: 1$ 进行实验比较, 结果表明(图 2), 当提取溶剂和茶叶 样品的比例为 $5: 1(\mathrm{~mL} / \mathrm{g})$ 时, 白茶、绿茶、花茶、乌 龙茶、红茶等不同茶类茶叶中的 7 种待测农药均可被 定量提取出来, 提取率令人满意, 且提取液中色素和 多酚类等干扰物质含量可以接受, 提取液易于净化 并不影响 HPLC-MS/MS 的检测. 当比例高于 5:1 时, 提取率基本不变, 且需要较多乙腈, 增加了成本. 综 合考虑上述各种因素, 本实验选取 5:1 作为提取溶剂 和茶叶样品的最佳比例.

$N$-丙基乙二胺、石墨化炭黑和 $\mathrm{C}_{18}$ 是 $\mathrm{QuEChERS}$ 方法中经常使用的固相萃取的固定相. 茶叶基质复 杂, 其提取液中含有部分色素、多酚类和脂肪酸等干

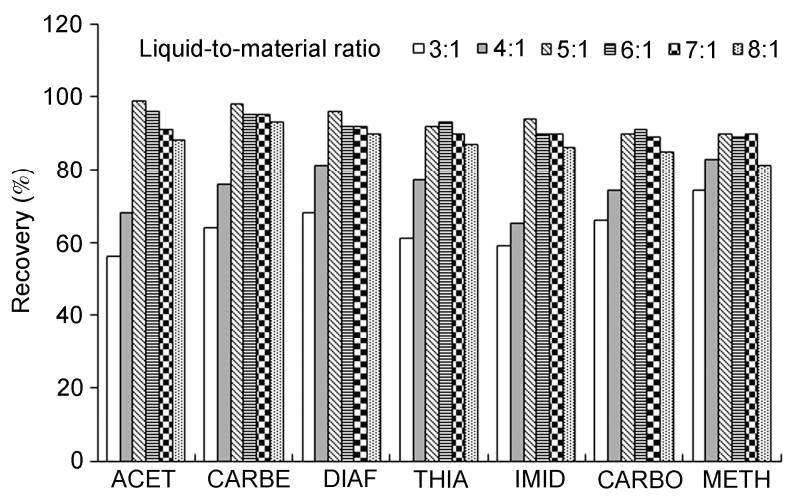

图 2 剂料比对茶叶中 7 种待测农药提取率的影响. 茶叶种 类: 红茶; 茶叶加标浓度: $0.1 \mu \mathrm{g} / \mathrm{g}$ (茶叶)
扰物质, $N$-丙基乙二胺能有效去除提取液中的脂肪 酸、碳水化合物、酚类和少量色素; 而石墨化炭黑能 去除色素、类胡夢卜素、固醇等杂质，但去除脂肪酸 的效果不佳 ${ }^{[22]}$. 李春艳 ${ }^{[36]}$ 考察了 $N$-丙基乙二胺和石 墨化炭黑的实际净化效果，发现 $N$-丙基乙二胺可去 除 90\%以上的脂肪酸和 50\%左右的色素，石墨化炭 黑可去除 90\%以上的色素，但对脂肪酸的净化能力 仅有 $10 \%$ 左右. 对于一些含色素高的样品, 将两者联 合使用, 效果明显, 可净化绝大多数的色素和脂肪酸. 但使用石墨化炭黑的缺点是其具有强吸附性，在去 除色素的同时也会吸附部分待测农药 ${ }^{[36]}$, 所以其使 用量的控制十分重要.

本实验利用 $N$-丙基乙二胺去除茶叶提取液中的 脂肪酸、酚类等杂质，利用石墨化炭黑去除茶叶提取 液中色素. 由于不同种类茶叶的发酵程度不同，乙腈 提取液的基质也存在差别，特别是色素含量的不同. 发酵时间越长的茶叶, 提取液中的色素含量越多. 在 实验中，我们适当增加了石墨化炭黑的用量以保证 各种不同茶叶提取液中的色素都能得到有效去除. 同时, 为了减少石墨化炭黑对待测农药特别是多菌 灵(石墨化炭黑对多菌灵有较强的吸附效果)的吸附, 在实验过程中我们加入少量甲苯溶液以防止石墨化 炭黑对待测农药特别是多菌灵的吸附，提高待测农 药的回收率, 因为已有研究报道在茶叶提取液中适 当加入一些有机溶剂可减少石墨化炭黑对农药残留 的吸附 ${ }^{[37]}$. 实验结果表明, 在茶叶提取液中加入一定 量的甲苯可有效降低石墨化炭黑对待测农药特别是 多菌灵的吸附. 但是, 添加过多的甲苯会使得待测液 的极性偏低, 对随后的 HPLC-MS/MS 测定影响较大. 我们以多菌灵为代表对甲苯的加入量进行了优化, 实验结果(图 3)表明, 在 $1.5 \mathrm{~mL}$ 的各类茶叶提取液中 加入 $0.15 \mathrm{~g} \mathrm{~N}$-丙基乙二胺、 $0.15 \mathrm{~g}$ 石墨化炭黑以及 0.5 $\mathrm{mL}$ 的甲苯, 可以有效去除茶叶提取液中的色素、多酚 类和脂肪酸等杂质，并保证 7 种目标农药包括多菌灵 均有良好的回收率, 满足 HPLC-MS/MS 的检测要求.

\section{2 方法的线性范围、重现性、灵敏度和检测限}

7 种农药纯标溶液的 HPLC-MS/MS 总离子流图, 以及各农药定量离子的色谱图如图 4 所示. 从图 4 可 知，在最佳分析条件下， 7 种农药在 $18 \mathrm{~min}$ 内实现基 线分离, 每一种待测农药的定量离子色谱图峰型较 好, 无明显干扰峰. 为了获得 7 种待测农药校准曲线, 


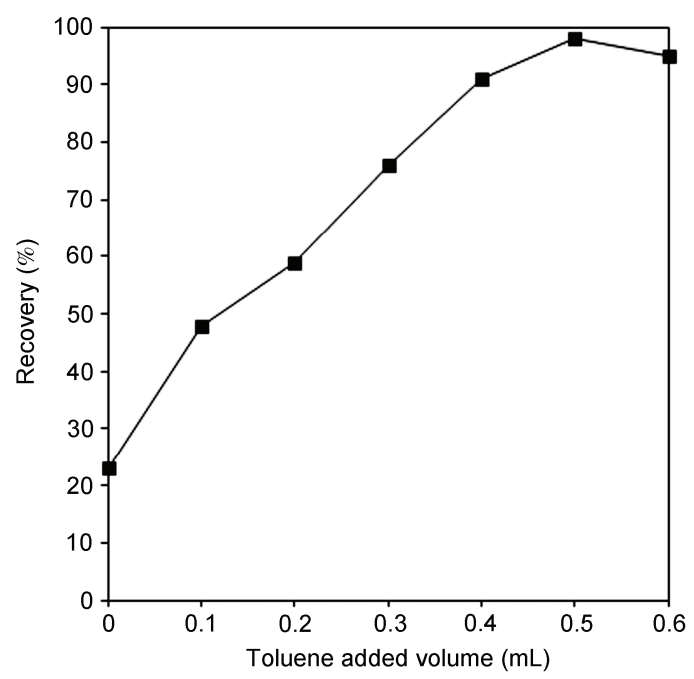

图 3 茶叶提取液中甲苯的加入量对多菌灵回收率的影响. 茶叶种类: 红茶; 茶叶加标浓度: $0.1 \mu \mathrm{g} / \mathrm{g}$ (茶叶). QuEChERS 法条件: 提取液 $1.5 \mathrm{~mL}, N$-丙基乙二胺 $0.15 \mathrm{~g}$, 石墨化炭黑 $0.15 \mathrm{~g}$

我们测定了一系列不同浓度的混合标准溶液, 其标 准曲线的线性方程、线性范围及其检出限见表 2 .

从表 2 可知, 色谱峰面积与浓度之间呈良好的线 性关系, 线性相关系数在 0.9937 0.9999 之间, 啶虫 脒、吡虫啉、噻虫嗪、多菌灵、克百威、灭多威和丁 醚脲的线性浓度范围分别为 $0.4 \sim 20 、 2.5 \sim 100 、 2.0 \sim$ 100、0.5 20、0.5 20、5.0 250 和 $1.0 \sim 50 \mathrm{ng} / \mathrm{mL} .7$ 种 农药的 IUPAC (国际纯粹与应用化学联合会)法检测 限(LOD, 以 3.84 倍空白溶液信号标准偏差计算)和定 量限 (LOQ, 以 10 倍空白溶液信号标准偏差计算)分 别为 $0.1 \sim 0.5$ 和 $0.26 \sim 1.3 \mathrm{ng} / \mathrm{mL}$. EURACHEM (欧洲分 析化学联合会)法 LOQ (定义为待测目标物的信号的 相对标准偏差 $\leqslant 10 \%$ 时待测目标物的最低浓度)为 $0.3 \sim 1.3 \mathrm{ng} / \mathrm{mL}$.

\section{3 方法验证}

为了验证方法的可靠性, 我们进行了加标回收 率实验, 在称量好的茶叶粉末样品中加入不同浓度 的标准品溶液 $1 \mathrm{~mL}$, 轻摇离心管, 使茶叶与标准溶 液混合均匀, 过夜, 使茶叶充分吸收标准品溶液. 第 二天用缓和 $\mathrm{N}_{2}$ 气流吹干茶叶后, 利用所建立的方法 分析检测加标茶叶样品, 每个加标水平重复测试 3 次. 回收率实验的结果见表 3 . 从表 3 可知, 3 种不同加标 水平的回收率值为 $86 \%$ 101\%, 3 次重复实验的日内

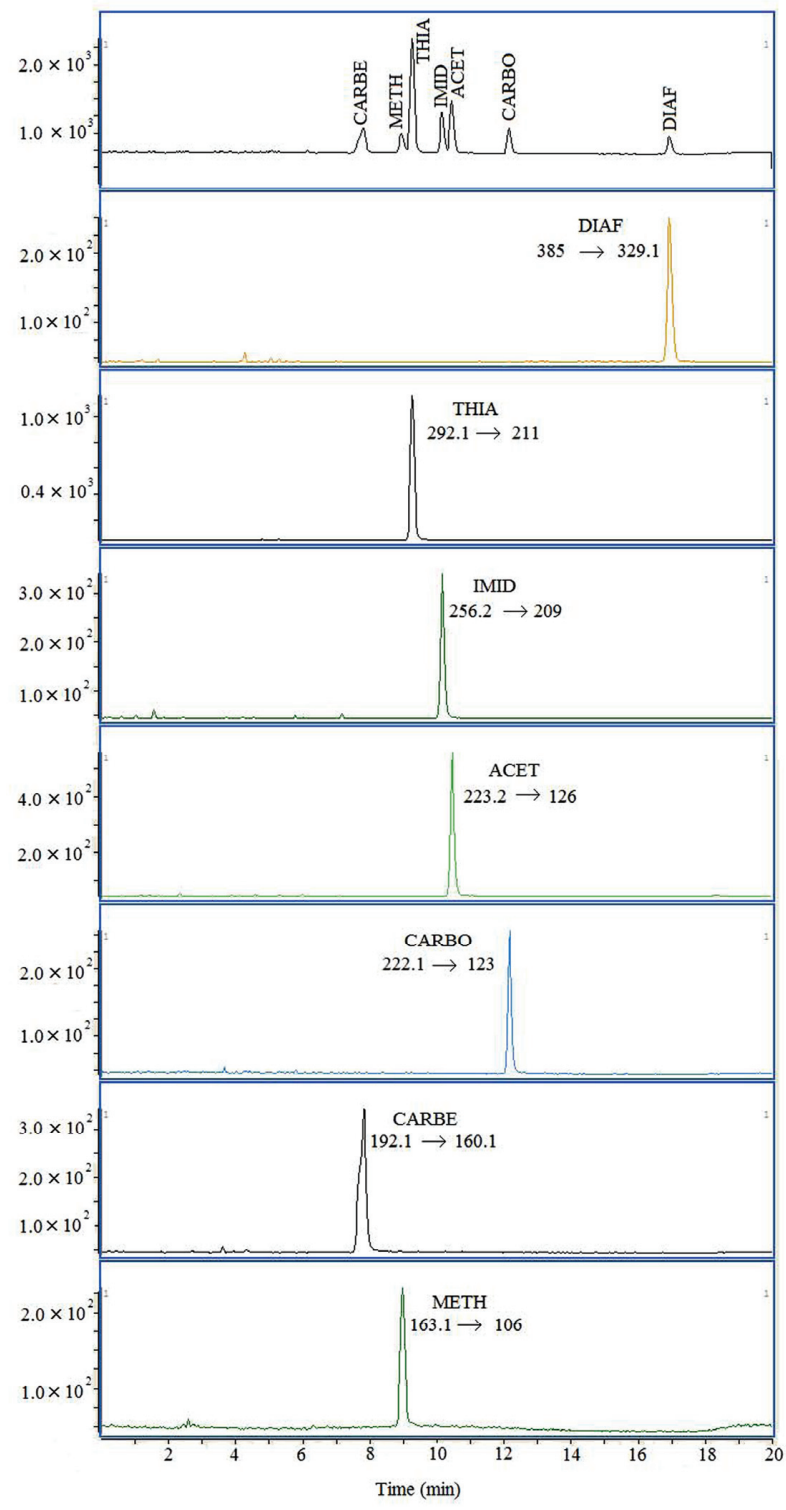

图 47 种待测农药(啶虫脒、多菌灵、丁醚艮、噻虫嗪、吡 虫啉、克百威和灭多威)混合标液的总离子流图, 和茶叶介质 中 7 种待测农药的总色谱图. 啶虫脒和多菌灵的浓度为 $20 \mathrm{ng} / \mathrm{mL}$, 吡虫啉和灭多威的浓度为 $50 \mathrm{ng} / \mathrm{mL}$, 丁醚脲和克 百威的浓度为 $10 \mathrm{ng} / \mathrm{mL}$, 噻虫嗪的浓度为 $100 \mathrm{ng} / \mathrm{mL}$

相对标准偏差(RSD, $n=3)$ 为 $2 \% \sim 6 \%$, 日间 $\operatorname{RSD}(n=3)$ 为 $2 \% \sim 8 \%$.

\section{4 实际样品的检测与分析}

采集福建省市场上的茶叶样本 30 份, 其中泉州 10 份、漳州 5 份、宁德 5 份、南平 5 份、福州 2 份、 三明 1 份、龙岩 1 份和厦门 1 份, 基本上覆盖了福建 
表 27 种待测农药的标准曲线的线性方程与检出限

\begin{tabular}{ccccccc}
\hline 组分 & 方程式 ${ }^{\mathrm{a})}$ & 相关系数 $\left(R^{2}\right)$ & 线性范围 $(\mathrm{ng} / \mathrm{mL})$ & $\mathrm{LOD}^{\mathrm{b}}(\mathrm{ng} / \mathrm{mL})$ & $\left.\mathrm{LOQ}^{\mathrm{c}}\right)(\mathrm{ng} / \mathrm{mL})$ & $\mathrm{LOQ}{ }^{\mathrm{d})}(\mathrm{ng} / \mathrm{mL})$ \\
\hline 啶虫脒 & $Y=210.8 X-13.49$ & 0.9998 & $0.4 \sim 20$ & 0.1 & 0.26 & 0.3 \\
吡虫啉 & $Y=47.98 X+29.41$ & 0.9999 & $2.5 \sim 100$ & 0.5 & 1.3 & 1.3 \\
噻虫嗪 & $Y=97.52 X+3.834$ & 0.9999 & $2.0 \sim 100$ & 0.2 & 0.52 & 0.7 \\
多菌灵 & $Y=392.4 X+18.33$ & 0.9996 & $0.5 \sim 20$ & 0.1 & 0.26 & 0.3 \\
克百威 & $Y=162.5 X-1.186$ & 0.9999 & $0.5 \sim 20$ & 0.1 & 0.26 & 0.3 \\
灭多威 & $Y=33.29 X+54.56$ & 0.9978 & $5.0 \sim 250$ & 0.5 & 1.3 & 1.3 \\
丁醚脲 & $Y=72.33 X-1.413$ & 0.9937 & $1.0 \sim 50$ & 0.5 & 1.3 & 1.3 \\
\hline
\end{tabular}

a) $X$ : 浓度 $(\mathrm{ng} / \mathrm{mL}) ; Y$ : 计数(峰面积); b) 仪器检测限(IUPAC 标准); c) IUPAC 标准; d) EURACHEM 标准 (RSD=10\%)

表 37 种待测农药在茶叶样品中的加标回收试验 $(n=3)$

\begin{tabular}{|c|c|c|c|c|}
\hline 组分 & $\begin{array}{c}\text { 加标水平 } \\
(\mathrm{ng} / \mathrm{mL})\end{array}$ & 回收率 (\%) & $\begin{array}{c}\text { 日内 RSD } \\
(\%)\end{array}$ & $\begin{array}{c}\text { 日间 RSD } \\
(\%)\end{array}$ \\
\hline \multirow{3}{*}{ 啶虫脒 } & 5 & 95 & 4 & 5 \\
\hline & 10 & 96 & 4 & 4 \\
\hline & 20 & 101 & 2 & 2 \\
\hline \multirow{3}{*}{ 多菌灵 } & 5 & 90 & 3 & 7 \\
\hline & 10 & 92 & 5 & 6 \\
\hline & 20 & 98 & 2 & 2 \\
\hline \multirow{3}{*}{ 丁醚衤 } & 5 & 94 & 5 & 5 \\
\hline & 10 & 96 & 5 & 5 \\
\hline & 50 & 96 & 4 & 4 \\
\hline \multirow{3}{*}{ 噻虫嗪 } & 5 & 86 & 6 & 8 \\
\hline & 10 & 91 & 4 & 5 \\
\hline & 50 & 92 & 2 & 2 \\
\hline \multirow{3}{*}{ 吡虫啉 } & 5 & 89 & 4 & 6 \\
\hline & 10 & 94 & 4 & 4 \\
\hline & 50 & 96 & 3 & 3 \\
\hline \multirow{3}{*}{ 克百威 } & 5 & 87 & 5 & 8 \\
\hline & 10 & 88 & 4 & 5 \\
\hline & 20 & 90 & 4 & 4 \\
\hline \multirow{3}{*}{ 灭多威 } & 5 & 86 & 5 & 6 \\
\hline & 10 & 86 & 4 & 5 \\
\hline & 100 & 90 & 3 & 3 \\
\hline
\end{tabular}

省的产茶区域及其主要茶叶品种, 其中乌龙茶 15 份、 红茶 5 份、绿茶 5 份、花茶 3 份和白茶 2 份. 用本实 验所建立的检测方法对实际茶叶样品中上述 7 种农 药残留进行分析检测.

实验结果表明, 在全部 30 份茶叶样品中, 有 9 份茶叶样品检出农药残留. 但是, 每份茶叶中只检出 7 种农药中的 1 种, 农药残留总检出率为 $30 \%$. 在检 出农药残留的 9 个样品中, 含吡虫啉残留的茶叶样品 有 5 份, 占总样品数的 $16.7 \%$, 检出浓度均在 0.05 $\mathrm{mg} / \mathrm{kg}$ 以下, 比国家标准限量值 $(\leqslant 0.5 \mathrm{mg} / \mathrm{kg})$ 小 1 个数 量级; 检出啶虫脒的茶叶样品有 3 份, 其中 1 份的检
测结果 $\geqslant 0.17 \mathrm{mg} / \mathrm{kg}$, 已超过农业部标准 $\mathrm{NY} / \mathrm{T}$ 288-2012《绿色食品茶叶》中规定的限量值 $(\leqslant 0.1$ $\mathrm{mg} / \mathrm{kg}$ ), 另外 2 份检出结果均小于 $0.1 \mathrm{mg} / \mathrm{kg}$; 检出灭 多威的茶叶样品有 1 份, 检测值 $0.20 \mathrm{mg} / \mathrm{kg}$, 远小于国 家标准 GB 2763-2014《食品安全国家标准 食品中农 药最大残留限量》中最大残留限量值 $(\leqslant 3.0 \mathrm{mg} / \mathrm{kg})$ 指标. 多菌灵、噻虫嗪、克百威残和丁醚嫝均未被检出.

\section{4 结论}

本文利用 QuEChERS 前处理技术结合 HPLC$\mathrm{MS} / \mathrm{MS}$, 建立了同时分析检测茶叶中啶虫脒、吡虫 啉、噻虫嗪、多菌灵、克百威、灭多威和丁醚脲 7 种 农药残留的分析方法. 优化了 QuEChERS 法的提取 和净化条件以及 HPLC-MS/MS 的检测条件. 所建立 的分析方法具有操作简单、快速和灵敏等优点, 可用 于同时检测茶叶中上述 7 种农药的残留, 7 种农药的 IUPAC 定量限为 $0.26 \sim 1.3 \mathrm{ng} / \mathrm{mL}$, 回收率为 $86 \%$ $101 \%$, 能够满足国内外茶叶标准对 7 种农药限量指 标的检测要求.

利用所建立的分析方法, 分析检测了采自福建 市场上的 30 个茶叶样品中 7 种农药残留情况. 在 30 份茶叶样中有 9 份检出农药残留, 总检出率为 $30 \%$. 但是 9 份茶叶中每份只检出 7 种农残中的 1 种. 其中 国家标准 GB 2763-2014 中有规定限量值的吡虫啉和 灭多威检出率共 $20 \%$, 但检出值较国家标准的限量 值小 1 个数量级, 不会对饮用产生质量安全问题. 啶 虫缕检出率为 $10 \%$, 其中 1 个样品略超过农业部标准 NY/T 288-2012《绿色食品 茶叶》中限量值 $(0.1 \mathrm{mg} / \mathrm{kg})$. 可见茶叶在生产过程中, 喷施吡虫啉、啶虫脒和灭多 威较为普遍, 且茶叶中的残留处于安全水平. 


\section{参考文献}

1 邓立刚, 李增梅, 郭长英, 赵善仓, 王玉涛, 毛江胜. 食品科学, 2011, 32: 221-224

2 吴刚，吴俭俭，赵珊红，郭方龙，陈浩，王力君，叶庆富. 中国食品学报, 2008, 8: 165-168

3 卿湘东, 吴海龙, 李元娜, 俞汝勤. 中国科学: 化学, 2010, 40: 1655-1663

4 US EPA. National Survey of Pesticides in Drinking Water Wells. Phase II Report. EPA 570/9-91-020, 1992

5 朴秀英、吕宁、林荣华、宗伏霖、刘学. 农药科学与管理, 2015, 36: 10-16

6 郭灵安, 雷绍荣, 仲伶俐, 胡莉, 梁立, 毛建霏. 食品研究与开发, 2012: 110-113

7 Muccio AD, Fidente P, Barbini DA, Dommarco R, Seccia S, Morrica P. J Chromatogr A, 2006, 1108: 1-6

8 侯如燕, 钱小三, 王孝辉, 市红正, 胡晋峰. 南京农业大学学报, 2013, 36: 123-127

9 路海燕, 李斌, 陈忠正, 高雄, 林晓蓉, 魏超田, 张媛媛. 食品科学, 2013, 34: 203-206

10 庄家祥, 傅建炜, 苏庆泉, 李建宇, 占志雄. 茶叶科学, 2009, 29: 154-158

11 赵秀霞, 张正竹, 胡祎芳, 苏婷, 侯如燕. 安徽农业大学学报, 2011, 38: 439-443

12 Kumar A, Verma A, Kumar A. Egypt J Forensic Sci, 2013, 3: 123-126

13 赵秀霞. 茶叶中噻虫溙残留检测方法及其在茶叶中的残留消解动态. 硕士学位论文. 安徽: 安徽农业大学, 2011. 7-9

14 张新忠, 罗逢健, 刘光明, 楼正云, 陈宗惁. 分析化学, 2014, 39: 1329-1335

15 Wang L, Zhao PY, Zhang FZ, Du FP, Pan CP. Ecotoxicol Environ Saf, 2012, 79: 75-79

16 Petroske E, Casida JE. Pestic Biochem Physiol, 2008, 58: 1-398

17 Commission Directive. EC 149/2008 amending Regulation (EC) No 396/2005 of the European Parliament and of the Council by establishing Annexes II, III and IV setting maximum residue levels for products covered by Annex I thereto. Official Journal of the European Union, 2008, 58: 1-398

18 Prasad R, Upadhyay N, Kumar V. Microchem J, 2013, 111: 91-96

19 Seccia S, Fidente P, Montesano D, Morrica P. J Chromatogr A, 2008, 1214: 115-120

20 Vichapong J, Burakham R, Srijaranai S. Talanta, 2013, 117: 221-228

21 朱宏斌, 胡银风, 姚射月, 沈春松, 岳鹏翔. 食品安全质量检测学报, 2015, 6: 1583-1589

22 张芬, 张新忠, 罗逢健, 陈宗惁, 孙威江, 刘光明, 楼正云. 分析测试学报, 2013, 32: 393-400

23 Lozano A, Rajski Ł, Belmonte-Valles N, Uclés A, Uclés S, Mezcua M, Fernández-Alba AR. J Chromatogr A, 2012, 1268: 109-122

24 Martínez-Domínguez G, Nieto-García AJ, Romero-González R, Frenich AG. Food Chem, 2015, 177: 182-190

25 Zhao PY, Wang L, Zhou L, Zhang F, Kang S, Pan C. J Chromatogr A, 2012, 1225: 17-25

26 孔志强, 董丰收, 刘新刚, 徐军, 龚勇, 单炜力, 郑永权. 分析化学, 2012, 40: 474-477

27 王连珠, 周昱, 黄小燕, 王瑞龙, 林子旭, 陈泳, 王登飞, 林德娟, 徐敦明. 色谱, 2013,31: 1167-1175

28 张志勇, 龚勇, 单炜力, 简秋, 沈燕, 刘贤进. 色谱, 2012, 30: 91-94

29 达晶, 王钢力, 曹进, 张庆生. 2015, 33: 830-837

30 李蓉, 储大可, 张朋杰, 高永清, 黄思允. 分析测试学报, 2015, 34: 502-511

31 Xu Y, Shou LF, Wu YL. J Chromatogr A, 2011, 1218: 6663-6666

32 Pang N, Wang T, Hu J. Food Chem, 2016, 190: 793-800

33 Mei M, Du ZX, Cen Y. Chinese J Anal Chem, 2011, 39: 1659-1664

34 刘飞, 陈永煊, 游飞明, 郑小严, 黄红霞. 食品研究与开发, 2012, 33: 144-146

35 王翔，宋国新，胡耀铭．质谱学报, 2008, 29: 97-100

36 李春艳. QuEChERS 法-色质联用技术在农药残留检测应用中的研究. 硕士学位论文. 陕西: 西安理工大学, 2010.23-28

37 Chen G, Cao P, Liu R. Food Chem, 2011, 125: 1406-1411 


\title{
Simultaneous determination of seven carbamates, neonicotinoids and diafenthiuron pesticide residues in tea by QuEChERS-HPLC-MS/MS
}

\author{
Lei Chen ${ }^{1,2}$, Liangmin Shangguan ${ }^{3}$, Fengfu $\mathrm{Fu}^{2^{*}}$ \\ 1 Institute for Agro-food Standards and Testing Technology, Shanghai Academy of Agricultural Sciences, Shanghai 201403, China \\ 2 Key Laboratory of Analysis and Detection for Food Safety of Ministry of Education; Collaborative Innovation Center of Food Safety Detection \\ Technology and Products, Fuzhou University, Fuzhou 350108, China \\ 3 National Tea Quality Supervision and Inspection Center (Fujian), Anxi 362400, China \\ *Corresponding author (email: fengfu@fzu.edu.cn)
}

\begin{abstract}
A novel method for the simultaneous determination of 7 carbamates, neonicotinoids and diafenthiuron pesticide residues (acetamiprid, imidacloprid, thiamethoxam, carbendazim, carbofuran, methomyl and diafenthiuron) in tea by QuEChERS pretreatment together with HPLC-MS/MS was described in this study. The optimum conditions of QuEChERS pretreatment and HPLC-MS/MS detection were investigated in detail in order to obtain the best performance of the method. The method reported in this study is simple and fast, it could be used to simultaneously determine above 7 pesticide residues in tea with an IUPAC LOQ of $0.26-1.3 \mathrm{ng} / \mathrm{mL}$ and a recovery of $86 \%-101 \%$. The success of this study provided a rapid, simple and reliable method for simultaneously detecting above 7 pesticide residues in tea.
\end{abstract}

Keywords: QuEChERS, HPLC-MS/MS, tea, carbamate pesticides, neonicotinoid pesticides, diafenthiuron doi: $10.1360 / \mathrm{N} 032015-00188$ 